\title{
Le monde rural québécois et la Politique nationale de la ruralité
}

\author{
Clermont Dugas 1 \\ Université du Québec à Rimouski
}

Les problèmes socio-économiques des localités et régions rurales qui durent maintenant depuis des décennies ont amené le gouvernement du Québec à mettre en place en 2001 sa Politique nationale de la ruralité. Cette dernière devrait permettre aux collectivités rurales de «léguer aux générations futures qui auront le goût de s'installer à la campagne, un territoire dynamique et prospère où il fait bon vivre» ${ }^{2}$. Compte tenu des problèmes existants, c'est un défi énorme que de vouloir rendre tout l'espace rural dynamique (au sens économique du terme) et prospère. Il y a donc lieu de se demander si la Politique sera à la hauteur de cet objectif. Nous essaierons ici de répondre à cette question en prenant en compte le contenu de la Politique ainsi que les caractéristiques du monde rural et des programmes et démarches antérieures de développement.

\section{Les principaux aspects de la Politique}

La Politique de la ruralité est présentée comme un cadre d'action devant susciter le dynamisme et la créativité des ruraux, leur permettant de se construire un avenir prometteur. Elle s'inscrit dans le prolongement de la Politique de soutien au développement local et régional de 1997 et prend fortement appui sur la théorie du développement local.

L'essentiel de la démarche gouvernementale retenue se résume à une injection de 90 millions de dollars sur cinq ans pour financer des projets initiés par la population dans plus de 1000 municipalités du Québec. Le territoire d'application est très diversifié allant des localités à faibles revenus et en forte dévitalisation aux petites villes et centres de services qui disposent d'un bon niveau de revenus et, dans bien des cas, bénéficient même d'une augmentation de population. Il appartient aux communautés rurales de mettre en place les projets qui vont modifier de façon durable leurs conditions de vie.
Quel que soit le degré de dynamisme et l'esprit d'innovation des résidents du monde rural, le budget de 90 millions sur cinq ans pour plus de 1000 localités constitue un levier économique insuffisant pour infléchir de façon significative les trajectoires de déstructuration qui existent dans une vaste partie du territoire rural. Sans tenir compte des coûts d'administration du programme, cela représente une moyenne annuelle de moins de $18000 \$$ pour chacune des localités ou $11.25 \$$ par personne. Cet argent va sans doute contribuer à créer des emplois temporaires et à réaliser de petits projets d'aménagement locaux tels que pistes cyclables, sentiers piétonniers, réparation de routes etc. Mais c'est nettement insuffisant pour assurer d'importantes créations d'emplois permanents, pour maintenir des services essentiels ou surtout pour corriger les problèmes structurels majeurs responsables du sous-développement. La faiblesse de ce budget est évidente quand on le compare à l'entente de développement CanadaQuébec de 258 millions \$ appliquée exclusivement à la Gaspésie et au Bas-St-Laurent pour la période 19681973 ou encore au 906.5 millions \$ investis dans quatre ententes de développement pour ces deux régions entre 1968 et 1993. On sait que la réalisation de ces ententes consécutives à une très forte mobilisation populaire est loin d'avoir réglé les problèmes de ces régions.

Cette Politique de la ruralité ressemble à bien des égards aux anciens programmes de création d'emplois du fédéral des années 1960, 1970 et 1980 tels que les travaux d'hivers, et les programmes d'initiative locale (PIL), d'aide à la création locale d'emplois (PACLE) et d'initiative jeunesse. Les projets qui étaient une émanation du dynamisme du milieu résultaient uniquement de l'initiative populaire et locale. Les sommes d'argent investies annuellement étaient aussi largement supérieures à celles de la Politique de la ruralité. Elles ont contribué à l'implantation d'infrastructures et à la 
création d'emplois temporaires, mais n'ont pu empêcher le dépeuplement rural et la perte de services.

\section{Le territoire d'intervention}

Le concept de «rural» retenu pour identifier l'espace d'application de la Politique est très sommaire et conduit à des ambiguïtés dans l'identification de certaines parties du territoire retenu. La caractérisation de cet espace fait aussi problème car elle tend à sous-évaluer la diversité du monde rural. Tout cela ne peut pas être sans conséquences sur les résultats de la Politique.

\section{Le concept de "rural " retenu pour identifier l'espace d'application de la Politique est très sommaire et conduit à des ambiguïtés dans l'identification de certaines parties du territoire retenu.}

En réalité, le terme «rural» n'est pas défini dans la Politique. On identifie plutôt comme rural le territoire qui se «situe essentiellement à l'extérieur des 31 grandes agglomérations urbaines du Québec ${ }^{3}$. On mentionne que ce territoire concerne plus de 1000 localités et plus de 1600000 personnes. Pour connaître l'espace concerné, il faut se référer à deux annexes ${ }^{4}$ où le territoire est découpé en fonction de trois catégories de MRC, celles à caractères rural, celles qui ont une agglomération de recensement et celles qui sont en partie dans les communautés métropolitaines.

Ce découpage conduit à des incohérences tant en regard des définitions de Statistique Canada que des appellations officielles du gouvernement du Québec. Ainsi, certaines municipalités qui ont le statut officiel d'urbaines pour Statistique Canada sont considérées rurales dans le cadre de la Politique. D'autres qui ont le statut de rural ou qui comprennent de fortes proportions de ruraux sont assimilées au monde urbain. Par ailleurs, des municipalités qui ont officiellement le statut de ville et qui sont régies par la loi des cités et villes font partie de l'espace rural dans le cadre de l'application de la Politique. C'est le cas pour de nombreuses petites villes comme La Sarre, Asbestos, Sainte-Anne-des-Monts, Amqui, Mont-Joli, Trois-Pistoles et Montmagny. Ces municipalités, même si elles sont de petite taille, ont des formes d'aménagement de type urbain et des fonctions urbaines.
L'objectif principal de la Politique de la ruralité est d'améliorer les conditions de vie et les perspectives d'avenir des ruraux. Or, ces dernières sont très diversifiées et dépendantes de nombreux facteurs à la fois d'ordres structurels et conjoncturels. Leur prise en compte s'impose pour mettre en place des moyens de solutions efficaces. Cependant, la politique est plutôt laconique sur ces aspects. Elle les résume en quelques considérations générales sur l'emploi, la migration, l'instruction et les services et en une esquisse typologique sommaire.

La typologie compte quatre grandes catégories de milieux ruraux: 1) ceux localisés dans l'environnement immédiat des centres urbains qui connaissent une forte croissance démographique; 2) ceux situés à une distance plus respectable de la ville, composés de localités relativement prospères mais dont la situation démographique et des services est diversifiée; 3) ceux localisés à la périphérie du territoire habité qui ont une situation socio-économique plus difficile et 4) ceux des centres de services qui contribuent à la dynamique des collectivités rurales qui les entourent.

Toutes les caractéristiques mentionnées dans cette classification sont justes mais elles ne traduisent qu'une partie de la réalité rurale et ne permettent pas de mesurer l'ampleur des problèmes qui s'y trouvent. De plus, cette typologie est sans utilité pour l'application de la Politique parce que les localités appartenant à chaque classe ne sont pas identifiées. En outre, la majeure partie des localités faisant partie de la première classe sont exclues du cadre d'application de la Politique puisqu'il s'agit des municipalités périurbaines appartenant aux agglomérations et régions métropolitaines de recensement. Par ailleurs, quelles que soient leurs caractéristiques, toutes les localités sont traitées sur le même pied dans l'application du programme, exception faite de la répartition du budget total entre MRC.

\section{Problématique, orientations et objectifs de développement}

Selon la terminologie utilisée dans le document, la Politique s'articule autour d' "un monde de défis» et de trois grandes orientations assorties chacune de trois objectifs. Les défis représentent les problèmes à solutionner. Le mot défi est très à propos, compte tenu de l'envergure des difficultés qui affectent une bonne partie 
de l'espace rural et de l'ampleur de la tâche à accomplir. Ces défis sont les suivants: un territoire à occuper, une économie à diversifier, des ressources à mettre en valeur, des gens à former, des services à assurer, une identité à préserver, un dynamisme à encourager, une intervention gouvernementale à adapter. Ce sont autant de titres qui évoquent des objectifs mentionnés dans des programmes et documents antérieurs ou dans des textes de revendication populaire.

\section{On fait, par exemple, une relation étroite entre le niveau de développement et le dynamisme des communautés. D'une part, on assimile toute la communauté à quelques individus peu dynamiques comme il y en a partout, même dans les villes et commu- nautés prospères, et d'autre part, on ignore tous les facteurs économiques, historiques, géographiques, biophysiques, culturels et sociaux qui ont généralement un impact déterminant sur le développement.}

L'exposé qui fait état des problèmes et tient lieu de problématique est très sommaire et contient certains propos qui méritent d'être précisés et nuancés. On fait, par exemple, une relation étroite entre le niveau de développement et le dynamisme des communautés. D'une part, on assimile toute la communauté à quelques individus peu dynamiques comme il y en a partout, même dans les villes et communautés prospères, et d'autre part, on ignore tous les facteurs économiques, historiques, géographiques, biophysiques, culturels et sociaux qui ont généralement un impact déterminant sur le développement. Ailleurs, on évoque la rationalisation dans la mise en valeur des ressources naturelles pour justifier les symptômes de la dévitalisation, mais un peu plus loin on mentionne que ces mêmes ressources peuvent être mises en valeur et favoriser l'émergence de produits locaux diversifiés et recherchés. On mentionne les statistiques faisant état de la sous-scolarisation des ruraux tout en étant silencieux sur l'important phénomène de migration des gens instruits. Dans un autre ordre d'idées, on écrit que sur le plan social, l'intervention de l'État a permis de stabiliser les revenus et de mettre les populations à l'abri des aléas de la conjoncture. S'il en était vraiment ainsi, comment doiton expliquer la forte dévitalisation qui affecte de nombreuses localités et l'élargissement des disparités de revenus? De tels propos qui, à tout le moins, demandent à être précisés apparaissent dans tout le chapitre 1 intitulé Le Québec rural... un territoire d'avenir qui tient lieu de problématique.

Les trois grandes orientations qui constituent autant de chapitres du document ne font pour l'essentiel que reprendre sous une formulation différente les énoncés qui figurent dans les défis. Il s'agit de:

- Stimuler et soutenir le développement durable et la prospérité des collectivités rurales;

- Assurer la qualité de vie des collectivités rurales et renforcer leur pouvoir d'attraction et

- Soutenir l'engagement des citoyens et citoyennes au développement de leur communauté et assurer la pérennité du monde rural.

Au total neuf objectifs découlent de ces orientations :

1) Diversifier l'économie, consolider et développer l'emploi et encourager l'esprit d'entreprise;

2) Accroitre la participation de la population à la gestion des ressources et à la mise en valeur de son territoire;

3) Intensifier l'acquisition du savoir, le développement des compétences et l'innovation;

4) Assurer le maintien et l'amélioration des services de proximité;

5) Améliorer et promouvoir le cadre de vie et accroître le sentiment d'appartenance;

6) Favoriser l'adaptation des politiques et des programmes gouvernementaux;

7) Promouvoir la place ainsi que le rôle des jeunes et des nouveaux arrivants dans le maintien des collectivités rurales dynamiques et le renouvellement des populations;

8) Tenir compte de la situation, des besoins et de l'apport particulier des femmes qui vivent en milieu rural; 
9) Renforcer la participation et l'engagement du monde rural et accentuer la complémentarité «rural-urbain».

Ce sont autant d'objectifs qui rallient sans doute tous les acteurs du développement et qui correspondent à la fois aux revendications traditionnelles des ruraux et aux orientations de développement qui figurent depuis des années dans des projets et programmes gouvernementaux, des plans stratégiques des CRCD et des ententes-cadre de développement. Diversification de l'économie, formation de la main-d'œuvre, participation populaire, complémentarité urbain-rural, etc. faisaient partie des objectifs du Bureau d'aménagement de l'Estdu-Québec (BAEQ) en 1966. La modulation de politiques et programmes gouvernementaux constituait l'un des volets de la Politique de développement régional de 1988.

La formulation répétée depuis plus de 30 ans de tous ces objectifs n'a pu empêcher la dégradation de la situation dans de nombreuses localités. Le véritable problème dans l'actuelle Politique de la ruralité se situe comme par le passé dans les moyens mis en place pour atteindre les objectifs poursuivis, que ce soit aux plans financier et organisationnel et aussi, dans une certaine mesure, dans la portée trop ambitieuse de certains de ces objectifs compte tenu du contexte en place.

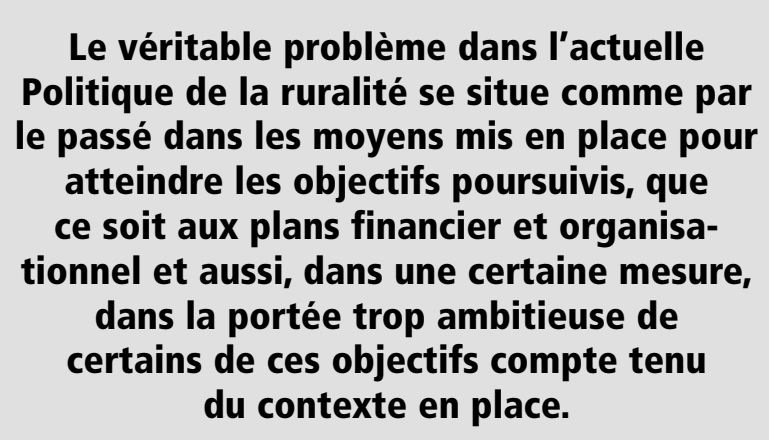

\section{L'administration de la Politique}

Les MRC sont responsables de l'application de la Politique. Par l'intermédiaire du pacte rural signé avec le gouvernement québécois, chacune reçoit une enveloppe budgétaire en fonction du nombre de ses municipalités rurales et de leurs problèmes de développement. C'est à la MRC qu'il appartient d'initier, encadrer et financer les projets de développement élaborés par les communautés locales.
Divers organismes comme les CLD, CRD et CAR sont considérés comme mandataires dans la mise en œuvre du programme. Enfin, un comité des partenaires de la ruralité est mis en place dans une perspective de concertation de différents organismes québécois dont Solidarité rurale qui se voit garantir un financement par le gouvernement jusqu'à 2005 et qui est considéré comme un interlocuteur privilégié.

Ce n'est donc plus aux technocrates des gouvernements fédéral et provincial, comme c'était le cas dans le passé, qu'il appartient d'élaborer, de réaliser et de contrôler des projets de développement mais aux élus locaux réunis au conseil de la MRC. Une telle approche s'inscrit dans le sens des revendications traditionnelles de décentralisation de nombreux intervenants régionaux. Mais la Politique a aussi ses droits à l'intérieur des MRC. Dans beaucoup d'entre elles, des zones de conflits existent entre secteurs urbain et rural. Le poids politique des localités y est fort inégal. Comment cela va-t-il se traduire dans l'application de la Politique? Cette situation est susceptible de provoquer un élargissement des écarts socio-économiques à l'intérieur du monde rural.

Même si, dans le texte de la Politique, on fait état de la volonté d'aider les localités les plus à problèmes, il n'y a pas de modalités d'application qui garantiraient des interventions - ou une «priorisation» de projets - orientées dans ce sens. Bien au contraire, l'extension du cadre d'application aux petites villes et centres de services qui disposent au départ d'une meilleure situation socioéconomique et d'un poids démographique et politique plus grand au sein des MRC, devrait logiquement les favoriser dans la distribution des investissements. En outre, les localités qui abritent les bureaux des MRC et CLD bénéficient en plus des retombées relatives à l'administration du programme. Dans ce contexte, il est difficile de concevoir comment les petites localités les plus marginalisées à tout égard, et qui ont le plus de besoins à combler, pourraient obtenir les investissements suffisants pour leur donner une nouvelle impulsion.

\section{Éléments d'appui de la politique}

Les chapitres 4, 5 et 6 de la Politique de la ruralité qui contiennent des moyens pour atteindre les objectifs sont pour l'essentiel un rappel des mesures gouvernementales existantes sous la responsabilité des divers ministères. Ce sont surtout des éléments de programmes qui concernent, à des degrés divers, le monde rural. Ils 
sont toutefois présentés en complémentarité avec la mesure spécifique à la Politique de la ruralité pour rappeler la diversité et sans doute l'envergure des moyens mis à la disposition des communautés rurales pour assurer leur épanouissement. Certains de ces programmes sont aussi bonifiés dans le cadre de la Politique.

Une trentaine de ministères et organismes, au moins 50 mesures et 24 programmes, politiques ou énoncés sont mentionnés dans la Politique. Cela peut donner l'impression d'un bel effort de concertation à l'égard du monde rural. Il s'agit en fait d'un inventaire de mesures existantes ou à venir appliquées de façon sectorielle selon la programmation de chaque organisme concerné.

\section{Une trentaine de ministères et organismes, au moins $\mathbf{5 0}$ mesures et $\mathbf{2 4}$ programmes, politiques ou énoncés sont mentionnés dans la Politique. Cela peut donner l'impression d'un bel effort de concertation à l'égard du monde rural. Il s'agit en fait d'un inventaire de mesures existantes ou à venir appliquées de façon sectorielle selon la programmation de chaque organisme concerné.}

Les mesures concernées sont très diversifiées et témoignent de la place omniprésente de l'État dans toutes les sphères d'activité de la société. Si certaines sont relativement nouvelles, d'autres existent déjà depuis assez longtemps soit sous leur nom actuel ou selon des variantes de d'autres appellations ou modalités différentes d'application de mesures antérieures. Certaines s'appliquent de façon spécifique au monde rural, d'autres davantage au monde urbain. Elles vont du programme d'aide à la mise en valeur des forêts privées et d'une stratégie globale de développement pour les produits du terroir à l'enfouissement des câbles de distribution d'Hydro-Québec, à une représentation féminine équitable au sein des conseils d'administration des CLD et CRD et au financement des universités en région.

Dans plusieurs cas, les mesures annoncées sont plutôt des intentions, des objectifs et des propositions que des engagements fermes. C'est notamment le cas du ministère de la Santé et des Services sociaux qui souhaite améliorer plusieurs aspects de la santé des individus, du ministère de l'Éducation qui favorise le maintien de l'école de village en regard de certains critères, du ministère de la Famille et de l'Enfance qui veut favoriser le soutien au rôle parental et du ministère des Transports qui propose de mettre à contribution les différents services de transport généraux ou spécialisés qui desservent déjà le territoire rural.

Des mesures applicables à l'ensemble du Québec sont susceptibles de favoriser davantage le monde urbain que rural. Il s'agit particulièrement d'éléments de la Politique familiale du ministère de la Famille et de l'Enfance, de programmes du ministère de la Culture concernant l'amélioration des équipements culturels et le renouvellement des expositions permanentes des institutions muséales, du programme Accès logis et des modalités de financement des cégeps et universités.

Certains aspects de la contribution du ministère des Affaires municipales et de la Métropole sont pour le moins discutables. L'organisme s'engage à investir $20 \%$ du budget des travaux d'infrastructure Canada-Québec et du programme Québec-municipalité en milieu rural. C'est un pourcentage inférieur à celui du nombre de ruraux au Québec. Par ailleurs, l'attribution récente de nouvelles responsabilités aux MRC à caractère rural est présentée comme un des moyens privilégiés pour favoriser la participation rurale à la réalisation de la Politique. Or, il n'y a rien dans ces nouvelles responsabilités qui constitue selon le gouvernement un «renforcement des MRC à caractère rural» pour susciter davantage la mobilisation des ruraux.

\section{Le discours gouvernemental}

Dans la Politique de la ruralité, les mesures gouvernementales ont cette particularité d'être diluées dans un texte qui insiste beaucoup sur le dynamisme et la mobilisation des ruraux et sur les interventions de l'État à leur égard mais qui est très laconique sur leurs contraintes au développement et sur les véritables causes de leur sous-développement. Ce discours gouvernemental comprend trois grandes composantes. L'une tend à démontrer que le gouvernement a beaucoup de sollicitude pour les ruraux, une deuxième semble vouloir justifier auprès de l'ensemble de la société québécoise, et surtout du monde urbain, les investissement gouvernementaux envers le monde rural et la troisième 
vise surtout à stimuler les énergies des ruraux pour qu'ils prennent en main leur développement.

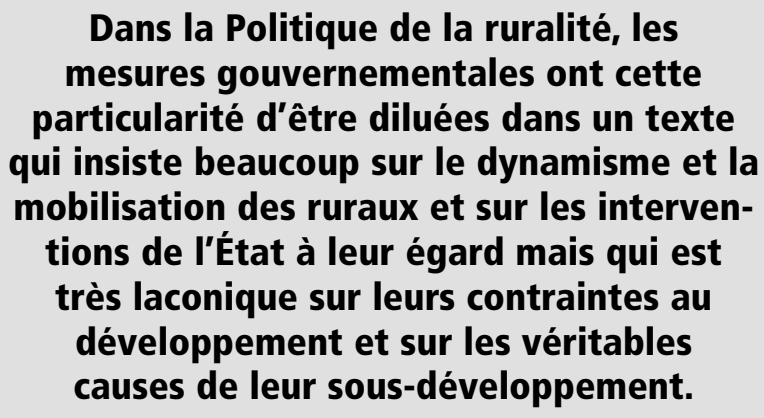

Dans la Politique de la ruralité, les mesures gouvernementales ont cette particularité d'être diluées dans un texte qui insiste beaucoup sur le dynamisme et la mobilisation des ruraux et sur les interventions de l'État à leur égard mais qui est très laconique sur leurs contraintes au développement et sur les véritables causes de leur sous-développement.

Le chapitre 1 de la Politique intitulé Le Québec rural... un territoire d'avenir est introduit par une présentation de la contribution du monde rural à l'économie du Québec et particulièrement à celle du Québec urbain. L'insistance est mise sur la valeur économique du monde rural plutôt que sur les nombreux problèmes économiques et sociaux qui affectent ce milieu de vie. Tout se passe comme si on voulait démontrer aux résidents des agglomérations urbaines qu'une intervention pour le monde rural est utile pour eux.

La troisième composante du discours gouvernemental fait appel au dynamisme et à la créativité des ruraux. C'est selon le gouvernement, à eux qu'appartient essentiellement la responsabilité de se bâtir un avenir prospère. Cet appel à la mobilisation constitue le principal fondement de la politique. Il va de soi que les ruraux doivent s'impliquer dans l'aménagement et le développement de leur milieu de vie. Mais ça, ils l'ont toujours fait et cette implication a pris de nombreuses formes durant les dernières décennies allant de la création de nombreux organismes pour le développement de l'emploi et des services à des luttes acharnées pour éviter la perte de services essentiels ou pour tout simplement pouvoir rester sur place.

Cette implication requise de la part des ruraux ne tient pas compte des multiples facteurs de marginalisation et de disparités sur lesquels les individus n'ont pas de prise. Les nombreux appels à la décentralisation provenant des régions ressources depuis au moins 40 ans ne sont qu'une pâle expression du sentiment d'impuissance qui habite leurs résidents en regard des multiples contraintes, contrôles, pressions, orientations et décisions qui émanent de l'extérieur.
Dans la Politique, le dynamisme des ruraux est considéré comme la principale cause du développement des localités prospères. "Le dynamisme de ces communautés fait en sorte que leur population s'accroît, se renouvelle et leur gamme de services s'améliore dans plusieurs secteurs ${ }^{4}$. On laisse aussi entendre que cette prospérité est à la portée de tous dans la mesure où la population fera preuve de dynamisme, d'imagination, d'audace et de créativité. Il est évident que le dynamisme est indispensable et qu'il peut être porteur de prospérité. Cependant, c'est ignorer les multiples efforts de reconversion économique et d'amélioration des conditions sociales réalisés par la plupart des communautés rurales depuis au moins trois décennies que d'en faire le principal facteur de développement. Le dynamisme et l'imagination manifestés par la population n'ont pas su empêcher la déstructuration et la dévitalisation de leur milieu de vie parce qu'il y a beaucoup d'autres facteurs en cause liés à la nature des ressources et des services disponibles, à la localisation géographique, à la taille démographique, au contexte régional et aux multiples décisions et événements qui viennent de l'extérieur.

À défaut de proposer des projets précis de développement, le gouvernement invite les gens à être créatifs, imaginatifs et audacieux, à se concerter, à se mobiliser, à s'approprier le développement, à relever des défis, à se doter d'une vision d'avenir, à s'ouvrir au défi du développement et à faire du réseautage entre municipalités rurales et urbaines. Le gouvernement tient aussi à valoriser les ruraux dans leur rôle de développeur. «En adoptant la politique nationale de la ruralité, le gouvernement fait confiance à la capacité des collectivités rurales à gérer leurs ressources et à raviver les forces de leur milieu». En fait, le gouvernement retourne aux résidents du monde rural la responsabilité de trouver des solutions à leurs problèmes comme si ce n'était pas ce qu'ils essaient de faire depuis longtemps. La confiance manifestée dans leur capacité de gérer leurs ressources a peu de signification pratique, étant donné la très étroite marge de manœuvre qu'ils ont en ce sens et les perspectives plutôt limitées de mise en valeur.

Les potentiels de développement sont survalorisés du simple fait de leur généralisation à l'ensemble du monde rural. Toutes les localités sont loin d'avoir les mêmes opportunités de développement et ne peuvent pas toutes se doter d'une vision d'avenir pleine de belles 
promesses. Il ne s'agit pas d'une simple question de détermination et d'engagement. Dans les faits, les inégalités existantes ne peuvent faire autrement que de conduire à un élargissement des disparités puisque tout le processus de développement attendu repose exclusivement sur les ressources et énergies locales.

\section{Conclusion}

Cette Politique de la ruralité tant attendue va incontestablement contribuer à améliorer de façon temporaire les conditions de vie d'un certain nombre de résidents du monde rural. Elle va aussi laisser des aménagements et des infrastructures dont l'utilisation va dépasser la durée de son application. Elle va sans doute contribuer à créer un certain nombre d'emplois permanents dans de nouvelles entreprises. Par ses modalités d'application, elle va contribuer à générer de nouvelles dynamiques politiques et sociales qui sont susceptibles également d'avoir des effets durables. Mais il est évident qu'elle est insuffisante pour ramener dans la voie de la prospérité et de la croissance démographique les centaines de municipalités qui vivent depuis des décennies un implacable processus de déstructuration. Il faut plus qu'un appel à la mobilisation et des promesses d'un avenir meilleur pour créer emplois et activités dans ces endroits défavorisés par un potentiel biophysique ingrat, une localisation à la marge du peuplement ou tout un contexte socio-économique défavorable. Les jeunes, les travailleurs sans emplois et les personnes âgées qui ont quitté ces localités ou qui s'apprêtent à le faire savent bien qu'il n'y a pas là d'emplois pour eux ni de services correspondant à leurs besoins et aspirations.

Par ses modalités d'application, elle va contribuer à générer de nouvelles dynamiques politiques et sociales qui sont susceptibles également d'avoir des effets durables. Mais il est évident qu'elle est insuffisante pour ramener dans la voie de la prospérité et de la croissance démographique les centaines de municipalités qui vivent depuis des décennies un implacable processus de destructuration.

\section{Notes et références}

1 Clermont Dugas est professeur à l'Université du Québec à Rimouski.

2 Politique nationale de la ruralité, p.1.

3 Idem, p.3.

4 Idem. Annexes ..., pp.54, 26 et 11.

4 Idem, p.8. 